\title{
Reflexión Crítica sobre la Administración del Desempeño laboral
}

\author{
Critical Reflection on Job Performance Management
}

Reflexão crítica sobre gestão de desempenho no trabalho

\section{ARTÍCULO GENERAL}

\section{Alexander Véliz Montero \\ Universidad César Vallejo \\ avelizm@ucvvirtual.edu.pe \\ https://orcid.org/0000-0002-3075-9793}

Recibido 01 de Septiembre 2021 | Arbitrado y aceptado 01 de Septiembre 2021 | Publicado en 02 Octubre 2021

\begin{abstract}
RESUMEN
En la presente se realizó una reflexión sobre el sistema de evaluación del manejo, haciendo énfasis en el valor y uso del Enfoque Participativo, donde el líder inmediato, el par, el subordinado, el comprador interno/externo es quien se encarga de evaluar empleando la metodología de competencias laborales. Según la práctica profesional personal aplicar dicha evaluación del manejo supuso un procedimiento transversal. Dicho suceso permitió que se transmita en el trabajo la meditación un sistema de Gestión del Manejo usado por varias multinacionales, y plantear la construcción de una Plataforma de Desarrollo Personal que haga más sencilla la gestión de información del manejo para aumentar la productividad y el aumento personal del trabajador en sus esferas de relación social. Con la meditación se lleva a cabo claves de la mejor práctica, para borrar las equivocaciones en la evaluación del manejo, en especial la subjetividad.

Palabras claves: Administración, Desempeño laboral, Competencias, Enfoque Participativo por Objetivos.
\end{abstract}

\section{ABSTRACT}

In the present, a reflection was made on the management evaluation system, emphasizing the value and use of the Participatory Approach, where the immediate leader, the peer, the subordinate, the internal / external buyer is the one in charge of evaluating using the methodology of labor competencies. According to personal professional practice, applying said management assessment involved a cross-sectional procedure. This event allowed the meditation of a Management Management system used by several multinationals to be transmitted at work, and to propose the construction of a Personal Development Platform that makes the management of management information easier to increase productivity and personal growth. of the worker in their spheres of social relationship. With meditation, keys of best practice are carried out, to erase mistakes in the evaluation of driving, especially subjectivity.

Keywords: Administration, Work performance, Competences, Participatory Approach by Objectives.

\section{RESUMO}

No presente, foi feita uma reflexão sobre o sistema de avaliação de gestão, enfatizando o valor e a utilização da Abordagem Participativa, onde o líder imediato, o par, o subordinado, o comprador interno / externo é o encarregado de avaliar utilizando a metodologia de competências laborais. De acordo com a prática profissional pessoal, a aplicação da referida avaliação de gestão envolveu um procedimento transversal. Este evento permitiu a meditação de um Sistema de Gestão de Gestão utilizado por várias multinacionais a ser transmitido no trabalho, e propor a construção de uma Plataforma de Desenvolvimento Pessoal que facilite a gestão da informação de gestão para aumentar a produtividade e crescimento pessoal. esferas de relacionamento social. Com a meditação, são realizadas chaves de boas práticas, para apagar erros na avaliação da condução, especialmente a subjetividade.

Palavras-chave: Administração, Desempenho no trabalho, Competências, Abordagem Participativa por Objetivos. 


\section{Introducción}

La presente reflexión es el resultado de haber revisado los procedimientos con los que se evalúa el manejo, y está orientada a destacar el valor y uso de llevar a cabo una Evaluación Participativa por Objetivos.

La Gestión del Funcionamiento se compone de normas y políticas, el método, el proceso de evaluación del manejo, el formato de evaluación, e instructivo (Valencia, 2002). Además, en la práctica se constituye de prácticas y pilares que incluyen la gestión de secuelas, red interna para comunicación continua respecto a los planes de acción y manejo.

La Evaluación del Funcionamiento refiere a un procedimiento que permite medir el funcionamiento del trabajador (Chiavenato, 2004). Así, el funcionamiento de un empleado está vinculado a la energía o esfuerzos que se gastan al ejecutar su trabajo así como el rendimiento o nivel de ejecución de las actividades que componen su trabajo (Martínez y Zapata, 2007). Este, se entiende, además, como el procedimiento mediante el cual se comunica al trabajador cómo debe llevar a cabo su labor, determinándose una estrategia de mejora continua (Byers y Rue, 2006)

Las competencias laborales son el grupo de conductas afectivas y sociales y las capacidades sensoriales, psicológicas, motoras y cognoscitivas que hacen posible realizar de forma idónea un rol, funcionalidad, labor o actividad (Valencia, 2005). Se puede decir también que las competencias laborales representan una incorporación de saberes, capacidades así como propiedades individuales primordiales en la consecución de un comportamiento definido de alusión predeterminado por la empresa o el área benéfico, en la que se base el proceso de comercio (Espinosa, 2011).

Cabe mencionar que son el líder, el propio trabajador, el par, el subordinado, el comprador interno y/o externo los que evaluarán.

Se define desempeño como "aquellas acciones o com- portamientos observados en los empleados que son relevantes para los objetivos de la organización", y que pueden ser medidos en términos de las competencias de cada individuo y su nivel de contribución a la empresa. Algunos investigadores argumentan que la deinición de desempeño debe ser 
completada con la descripción de lo que se espera de los empleados, además de una continua orientación hacia el desempeño efectivo. La Administración del desempeño, es deinida como "el proceso mediante el cual la compañía asegura que el empleado trabaja alineado con las metas de la organización”, así como las prácticas a través de las cuales el trabajo es deinido y revisado, las capacidades son desarrolladas y las recompensas son distribuidas en las organizaciones. Si bien diferentes estudios sobre indicadores de productividad y inancieros han demostrado que en las compañías en las que se implementan sistemas de administración del desempeño, los empleados han obtenido mejores resultados, que en las que no fueron utilizadas, los ad- ministradores deben ser conscientes de que cualquier falla de las organizaciones en adoptar una efectiva administración del desempeño es costosa, en términos de pérdida de oportunidades, actividades no enfocadas, pérdida de motivación y moral. Recientemente se ha descubierto que toda Administración del desempeño es un ciclo dinámico, que evoluciona

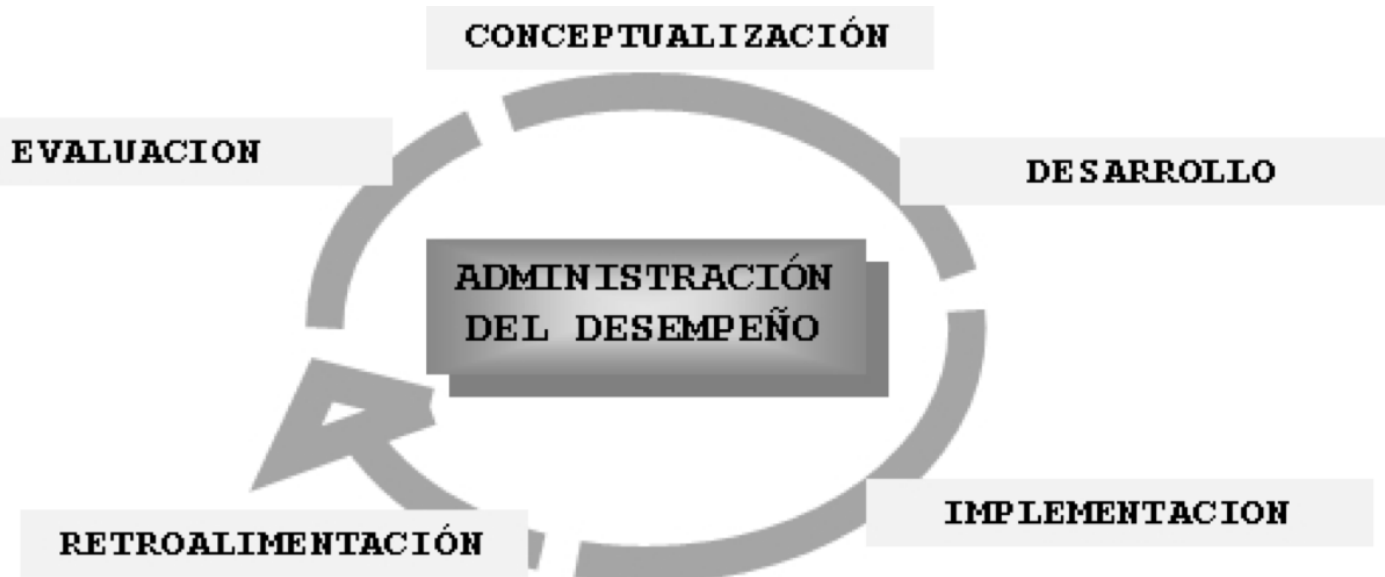

hacia la mejora de la compañía como un ente integrado. Como todo ciclo consta de etapas, las cuales pueden ser observadas en la figura 1.

Figura 1. Ciclo dinámico de la administración básica para implementación de un cambio.

La forma tradicional se realizaba mediante mecanismos informales de monitoreo del desempeño actual, seguido de sesiones de entre- namiento, revisiones periódicas del desempeño, entre otras; sin permitir al empleado tomar control de su propio desarrollo del desempeño. Retroalimentación es importante durante todo el proceso y también después de la evaluación para que el empleado sepa cuales son los puntos que debe reforzar para mejorar su desempeño, en miras de mejorar el desempeño integral de la compañía. 
Conceptualización es la fase en la que la empresa identiica el mejor rendimiento al cual desea dirigirse. Desarrollo es la fase en que la compañía examina dónde el rendimiento actual está variando en función de los niveles deseados, lo cual puede realizarse a través de un Sistema de Soporte Integrado del Desempeño. Implementación las medidas de desempeño para monitorear los indicadores especíicos de desempeño en todas las competencias y determinar cómo están respondiendo los objetivos.

\section{METODOLOGÍA}

La metodología utilizada consta de búsqueda de información de paper, revistas, análisis de aplicaciones actuales y páginas web especializadas para entender su funcionamiento, el nivel de desarrollo obtenido en la actualidad y cuáles son sus principales aplicaciones. Además, se emplean análisis de modelos estratégicos acordes para analizar el atractivo de las industrias en la adopción de la tecnología y así determinar a través de modelos de análisis de negocio cuáles son las industrias en que tendrá mayor incidencia.

La búsqueda de la información para la revisión de literatura se hizo consultando las siguientes bases de datos como son Proquest, Scopus y Google Scholar para encontrar revistas del más alto nivel científico. Asimismo, cabe mencionar que para la realización de lo mencionado se emplearon también palabras clave como como Administración, Desempeño laboral, Competencias, Enfoque Participativo por Objetivos.

Cada uno de estos descriptores se combinaron entre sí durante la búsqueda utilizando los operadores boléanos "and" y "or". Además, algunos de los criterios o filtros que se utilizaron para hacer muchos más específica la búsqueda y ser más precisos con los documentos encontrados en las diferentes bases de datos consultadas fueron: que sean artículos de revistas, y la antigüedad de la publicación que no sea mayor a 5 años.

Toda la información previa relacionada a los filtros aplicados y las especificaciones sirvieron como criterios de inclusión, es decir que sean artículos publicados en revistas científicas, que no excedan los cinco años de antigüedad, que la temática guarde relación con las variables de creatividad artística y tiempos de pandemia. Se excluyeron, en tanto, todos los documentos que no respetaran lo postulado así como aquellos que estaban incompletos o que tenían enlaces averiados.

\section{RESULTADOS Y DISCUSIÓN}




\subsection{Nociones básicas}

\section{Definiciones de la Evaluación del Desempeño Laboral}

"Lo podemos considerar como un procedimiento estructural y sistemático para medir, evaluar e inluir sobre los atributos, compor- tamientos y resultados relacionados con el trabajo, así como el grado de ausentismo, con el in de descubrir en qué medida es productivo el empleado y si podrá mejorar su rendimiento futuro, que permite implantar nuevas políticas de compensación, mejora el desem- peño, ayuda a tomar decisiones de ascensos o de ubicación, permite determinar si existe la necesidad de volver a capacitar, detectar errores en el diseño del puesto y ayuda a observar si existen problemas personales que afecten a la persona en el desempeño del cargo".

"Los principales objetivos de la evaluación del desempeño no pueden restringirse a un simple juicio supericial y unilateral del jefe respecto del comportamiento funcional del subordinado"; es necesario descender más profun- damente, localizar las causas y establecer perspectivas de común acuerdo con el evaluado.

Según Werther y Davis, "La Evaluación del Desempeño consti- tuye el proceso por el cual se estima el rendimiento global del empleado. Constituye una función esencial que de una u otra manera suele efectuarse en toda organización moderna”.

"Si se debe cambiar el desem- peño, el mayor interesado, el evalua-do, debe no solamente tener conocimientos del cambio planeado, sino también por qué y cómo deberá hacerse si es que debe hacerse".

Chiavenato, requiere que "Cuando un programa de evaluación del desem- peño está bien planeado, coordinado y desarrollado, normalmente trae benei- cios a corto, mediano y largo plazo. Los principales beneiciarios son, general- mente, el evaluado, el jefe, la empresa y la comunidad"

Mediante la evaluación del desempeño laboral, la organización, conoce los aspectos de compor- tamiento y desempeño que la empresa más valoriza en sus fun- cionarios. Da a conocer cuáles son las expectativas respecto al desempeño. A través de ella se anuncian las medidas que se van a tomar en cuenta para mejorar el desempeño (programas de entrenamiento, se- minarios, etc.) y las que el evaluado deberá tomar por iniciativa propia (autocorrección, esmero, atención, entrenamiento, etc.). 
La evaluación le da al trabajador la oportunidad para hacer autoevaluación y autocrítica para su autodesarrollo y autocontrol. Con ella se estimula el trabajo en equipo y se procura desarrollar las acciones pertinentes para motivar a la persona y conseguir su identificación con los objetivos de la empresa. Es un instrumento para mantener una relación de justicia y equidad con todos los trabajadores. Estimula a los empleados para que brinden a la organización sus mejores esfuerzos y vela por que esa lealtad y entrega sean debidamente recompensadas. Atiende con pron- titud los problemas y conlictos, y si es necesario toma las medidas disciplinarias que se justifican. Estimula la capacitación entre los evaluados y la preparación para las promociones.

Según Chiavenato. La evalua- ción del desempeño es una apreciación sistemática del desempeño de cada persona en el cargo o del potencial de desarrollo futuro.

Según Gibson. La evaluación del desempeño es un proceso sistemático mediante el cual se evalúa el desempeño del empleado y su potencial de desarrollo de cara al futuro.

Según Baggini. La evaluación del desempeño es el proceso por el cual se estima el rendimiento global del empleado.

La mayor parte de los empleados procura obtener retroalimentación sobre la manera en que cumple sus actividad y las personas que tienen a su cargo la dirección de las labores de otros empleados deben evaluar el desempeño individual para decidir las acciones que deben tomar.

\section{Beneficios de la Evaluación del Desempeño}

La evaluación del desempeño ayuda a implantar nuevas políticas de compensación, mejora el desempeño, refuerza la toma decisiones de ascensos o de ubicación, permite determinar si existe la necesidad de volver a capacitar, detectar errores en el diseño del puesto y ayuda a observar si existen problemas per- sonales que afecten a la persona en el desempeño del cargo. La evaluación del desempeño no puede restrin- girse a un simple juicio supericial y unilateral del jefe respecto del com- portamiento funcional del subordinado(11); es necesario descender más profundamente, localizar las causas y establecer perspectivas de común acuerdo con el evaluado.

Si se debe cambiar el desempeño, el mayor interesado, el evaluado, debe no solamente tener conocimientos del cambio planeado, sino también por qué y cómo deberá hacerse 
si es que debe hacerse. La evaluación del desempeño no es un in en sí misma, sino un instrumento, medio o herramienta para mejorar los resultados de los recursos hu manos de la empresa.

Para alcanzar ese objetivo básico y mejorar los resultados de los recursos humanos de la empresa, la Evaluación del Desempeño trata de alcanzar estos diversos objetivos intermedios: La vinculación de la persona al cargo, entrenamiento, promociones, incentivos por el buen desempeño, mejoramiento de las relaciones humanas entre el superior y los subor- dinados, autoperfeccionamiento del empleado, informaciones básicas para la investigación de Recursos Humanos, estimación del potencial de desarrollo de los empleados, estímulo a la mayor productividad, oportunidad de conocimiento sobre los patrones de desempeño de la empresa, retroalimentación con la información del propio indi- viduo evaluado, otras decisiones de personal como transferencias, gastos, etc.

Los objetivos fundamentales de la evaluación del desempeño pueden ser presentados en tres fases: Permitir condiciones de medida del potencial humano en el sentido de determinar su plena aplicación. Permitir el tratamiento de los Recursos Humanos como un recurso básico de la organización y cuya productividad puede ser de- sarrollada indeinidamente, depen- diendo, por supuesto, de la forma de administración. Proporcionar oportunidades de crecimiento y condiciones de efectiva partici- pación a todos los miembros de la organización, teniendo presentes por una parte los objetivos organizacionales y por la otra, los objetivos individuales.

El director obtiene los siguientesbeneicios con la evaluación, al constatar mejor el desempeño y el comportamiento de los subordina- dos, teniendo como base variables y factores de evaluación y, principalmente, contando con un sistema de medida capaz de neutralizar la subjetividad. Tomar medidas con el in de mejorar el comportamiento de los individuos. Alcanzar una mejor co- municación con los individuos para hacerles comprender la mecánica de evaluación del desempeño como un sistema objetivo y la forma como se está desarrollando éste. Planificar y organizar el trabajo, de tal forma que podrá organizar su unidad de manera que funcione como un engranaje.

El administrador, con la evaluación, señala con claridad a los individuos sus obligaciones y lo que espera de ellos; programa las actividades de la unidad, dirige y controla el trabajo y establece las normas y procedimientos para su ejecución. Además, invita a los 
individuos a participar en la solu- ción de los problemas y consulta su opinión antes de proceder a realizar algún cambio.

El mayor problema evidente en las Evaluaciones del Funcionamiento en la práctica laboral, y basado en la experiencia ejerciendo evaluaciones, es sin duda la Subjetividad con que se llevan a cabo y los efectos que suponen en los colaboradores por factores como la injusticia e inequidad.

Conforme con la práctica profesional, se crean modelos de evaluación del desempeño de corte transversal: a los 3 meses, 6 meses, al año. Estos se orientan a generar aumentos en la productividad de las personas. Numerosas empresas a nivel multinacional llevan a cabo esta Evaluación de Desempeño a modo de sistema y no como proceso, lo que supone una meta con ambición que a la vez implica ir a buen puerto en la misión de que se aumenten los índices productivos. No obstante, no se toman en consideración mecanismos que busquen motivar hacia la acción de un colaborar en sus medios de interacción social, para un desarrollo efectivo a nivel personal, así como social, profesional y familiar.

Cada procedimiento aporta un anillo en la cadena del desarrollo del entendimiento en funcionamiento. Los procedimientos que existen actualmente para detectar el funcionamiento de los individuos se exponen a continuación:

1. Método de Escala Gráfica: es una tabla de doble entrada que indica hasta diez factores para evaluar, con hasta cinco grados de variación, definiciones, De esta forma el que tiene un grado mayor es el que más desempeño presenta. Las equivocaciones se relacionan con la generalización, superficialidad y subjetividad.

\section{Método de Evaluación Enfoque Participativo por Objetivos}

3. Método de Incidentes Críticos: Está basado en los incidentes que ocurran (sean de éxito o fracaso). La equivocación se vincula a la información parcializada, no realiza análisis de desempeño que otorgue datos sobre los hábitos de las conductas del colaborador.

4. Método de Investigación de Campo: Se desarrolla, por el experto, un formulario desagregado en cuatro fases: entrevista inicial, entrevista de análisis 
Alexander Véliz Montero

complementario, planeación de las medidas y acompañamiento posterior a las respuestas, indicando en el documento los criterios del superior respecto a su colaborador (Chiavenato, 2004). La no participación del empleado y la lentitud son errores en este método.

5. Método de selección Forzada: Se evalúa en bloques de frases descriptivas sobre el comportamiento en el trabajo lo que se da en forma forzada. En cada bloque, la persona que está siendo evaluada elige la frase que mejor describe su desempeño y la frase que menos lo hace.

6. Método Listas de Verificación: Se realiza una revisión a cargo del jefe respecto a las características pre identificadas y en lista que debe suplir el empleado. 'Es una simplificación del método de escala gráfica’. (Chiavenato, 2004)

Por su utilidad, el método a detallar es la Evaluación Enfoque Participativo por Objetivos. Esta se opone al $100 \%$ a entregar un formulario, que se sabe el empleado no ha visto nunca con factores de evaluación, del que no tiene conocimiento y que su superior llenó sin estar calificado como evaluador (Puchol L. , 2000).

\subsection{Evaluación del Desempeño laboral como parte del Sistema de Administración del Desempeño.}

Los objetivos que están ligados a las aplicaciones:

- Asegurar la implementación de la estrategia.

- Introducir una cultura del desempeño

En el momento que se quiere generar una manera innovadora de realizar las cosas de forma independiente en la entidad, se requiere trabajar en prácticas, principios y valores tanto de jefes como de colaboradores.

El tema de valores queda esclarecido en, por ejemplo, gremios de Japón. En estos, si se presenta un contexto complejo, llevan a cabo el pliego de ofrecimientos, algo como de los 12 retardos en la gestión, ofrecen minimizar a 8 retardos (Kasuga, 2012). Al realizar un contraste con los gremios de América del Sur se identifica como producto un comportamiento dirigido a hacer pliego de peticiones. 
El sistema de administración del desempeño amerita que se restructure o produzca una cultura orientada al alto desempeño incluyendo principios y valores definidos por los directivos que guarden relación con la visión y misión de la entidad. Tal como:

- Trabajamos y ganamos en Equipo.

- Nuestra Responsabilidad es Individual.

- Las Personas son nuestra Ventaja Competitiva.

- La Retroalimentación en el trabajo es nuestra ventaja en la gestión humana.

- Valoramos y respetamos a las ideas innovadoras

- Mi compromiso es hacer lo mejor que puedo en cada situación.

- Ofrecemos soluciones a los problemas

- Nuestro enfoque organizacional es la satisfacción del comprador.

- Somos personas involucradas con nuestra organización.

- Somos idealistas, soñadores y llevamos los sueños a la verdad.

- Somos ganadores, vendemos resultados no la época

Un costo particular es el que se expresa como la fuerza gremial del emigrante que quiebra los obstáculos e incrementa divisas cuando manda dinero a sus familiares a modo de reconocimiento, devolviendo lo que recibió en vida (Cornejo, 2014). Esto no ocurre en civilizaciones como las de Europa o Norteamérica.

\subsection{Formulación de objetivos y metas en cascada (Puchol, 2003) deben} ser:

a) Entablados entre jefe y empleado y en cascada descendente que indica que va desde un nivel más alto en la jerarquía hacia uno inferior inmediato (Ej. presidente a vicepresidente, vicepresidente a director, director a gerente, gerente a jefe, jefe a su supervisor, jefe a su analista; supervisor a su asistente, etc.); 
Alineados al plan de negocios, planificación estratégica, requerimientos de los clientes externos e internos;

Ajustados a la dinámica del entorno organizacional; Deben ser Asumidos con responsabilidad individual.

La 4A son producto de las experiencias laborales:

b) El documento de establecimiento de metas acordadas no es un formulario palpable, sino, más bien una estrategia psicológica que fija el compromiso de cumplir metas y objetivos.

c) Asignar recursos y medios para la consecución de las metas planteadas.

d) Apegarse a los valores y principios de la organización, el sentido de la pertenencia a una cultura de desempeño de parte del colaborador y su jefe.

e) Continuo monitoreo de los resultados para las reuniones informales de orden mensual o bimensual como para las revisiones del desempeño formales de orden semestral.

f) Retroalimentación descendente (jefe - empleado) y ascendente (empleado jefe). Esta última retroalimentación es una de las claves para el éxito de la evaluación, porque fomenta y modela la cultura de alto desempeño. La retroalimentación usa a la comunicación continua del desempeño como el camino formal hacia la cumbre.

La Comunicación continua del Desempeño y la Planificación Estratégica son pilares de gran relevancia en la Administración del Desempeño a nivel operativo, administrativo y gerencial.

La cultura de desempeño es un trabajo que se forja diariamente mediante la gestión, planificación y en las prácticas comunicativas. A través de las decisiones y planes, el nivel jerárquico va a fomentar esta cultura organizacional a la par que los colaboradores la robustecerán con sus conductas y acciones. Es preciso que se respete el sistema de Administración del Desempeño organizacional y sus mejores prácticas de gestión tanto de jefes como de empleados. Se debe mantener la coherencia entre lo que se piensa y lo que se hace; así, impulsos y afinidades personales son lo opuesto a una base de toma de 
decisiones, de consecuencias para las personas; EPPO observa lo planeado y lo ejecutado, mira hacia el futuro no se ancla en el pasado.

\subsection{Entablar un proceso ambicioso y consistente para la Gestión del Manejo personal y de equipo}

El Sistema de Administración del Desempeño se conforma por políticas, proceso de establecimiento de objetivos, de metas; procedimiento de evaluación formal e informal, prácticas actitudinales del subordinado y su superior, con la más enorme consistencia y precisión. El desempeño de las personas, de conjuntos, por funciones, o niveles necesita de un manejo diario mediante un autocontrol. Así, al finalizar el mes se emplea para medir los indicadores de gestión, que deben estar relacionados a los objetivos. Tanto el jefe como el colaborador van a analizar la información en las reuniones informales cada mes o 2 meses, identificando aciertos y desaciertos así como las soluciones para estos últimos. A los 6 meses se llevan a cabo las revisiones formales del desempeño, en las que, a través de un dialogo bidireccional y presentación de pruebas de los interactuantes, el jefe va a asignar una calificación formal al manejo y gestiona los efectos como compensaciones, planes de desarrollo o retención, consejería, etc.

Las elecciones están a cargo de los dirigentes, no solo definen el triunfo económico de la entidad o su fracaso, sino además el impacto de esta en la raza humana a extenso plazo (Sanders, 2009).

\subsubsection{Los beneficios puntuales para la organización son los siguientes.}

\section{a) Beneficios para el área Estratégica:}

- Desarrollo y aplicación de una Estrategia Mejorada a través de personas enfocadas, alineadas entre funciones.

- Comunicación más efectiva de lo estratégico,

\section{b) Beneficios Culturales:}


La era apremia para realizar otra revolución en el sitio de trabajo, una dirigida por la cultura y la sinergia, girando por los ingresos y por la paz de los individuos (Sanders, 2009).

- Una cultura se forma por medio de la existencia de prácticas, una costumbre es un hábito, es la repetición de un comportamiento en un corto tiempo, tales como un mes constante.

- Una cultura hacia el elevado manejo 'cultura para personas que gozan comprometerse con su papel y exceden en sus resultados' (Cornejo, 2014). Ejemplo una vez que se elabora el desayuno el cerdito se compromete por esa razón consumimos el jamón, la gallina se implica por esa razón consumimos los huevos.

- A diario consumar con las prácticas conductuales de empleados, equipo.

- Enfoque hacia la satisfacción del comprador.

- Trascendencia, que el trabajador sea un mejor ser humano, un emprendedor con espíritu de desarrollo

\section{c) Beneficios que el Sistema de Administración del Desempeño brinda a las personas:}

- Una metodología robusta para establecer de metas, monitoreo, revisión formal del desempeño, identificación fortalezas, brechas, amenazas y desarrollo de habilidades necesarias.

- Identificación de Personas Estratégicas, que se convierte en una de las fuentes claves de la información de RH para el desarrollo de Planes de Carrera.

- Identificación de personas que exceden, que logran, que no logran las metas. En conjunto con las consecuencias que correspondan, como son: reconocimiento, premio, retención; y para quienes no logran: entrenamiento y asesoramiento.

- Prácticas mejoradas de los líderes. 
Las personas que llevan adelante la evaluación pueden ver la amplitud de la práctica convertida en una inmensa fuente de satisfacción y orgullo. La orientación hacia la evaluación de resultados crea cambios; evaluar e informar sobre los resultados es importante en las ocupaciones y procesos de la organización (Richard Swanson; Elwood Holton, 2002).

El enfoque se 'caracteriza por la autoevaluación, autodirección de los individuos, más grande colaboración del empleado en su proyecto de desarrollo personal, concentrando en el futuro y en el mejoramiento constante del desempeño' (Chiavenato, 2004). Su base es el cumplimiento de fines por medio del logro de metas ambiciosas que integran las competencias genéricas de la organización y concretas del puesto, su espíritu es el trabajo en valores culturales organizacionales. Haciendo un trabajo en los valores y un correcto sistema de Gestión del Manejo con indicadores de administración específicos e por igual ambiciosos, se puede modificar la manera de confrontar las situaciones en el trabajo, en el núcleo familiar, en la sociedad, en la sociedad y ser personas integralmente competitivas.

Los periodos de una evaluación del manejo gremial (Chiavenato, 2004) son:

\subsection{Propuesta para que Administración del Desempeño formalice el Desarrollo a través de la Plataforma de Crecimiento Personal.}

Las organizaciones precisan de un aumento en sus índices de productividad, esto llevado a un plan resulta perfecto, no obstante, no es lo único importante. La propuesta así, es diseñar una herramienta propia para la entidad, que haga posible que se trabaje en el desarrollo del personal basado en los resultados de las evaluaciones del desempeño permanente y/o de proyectos, brechas de competencias genéricas y/o específicas.

Entonces, es más que un curso para los trabajadores sino que se trabaja en una Plataforma de Crecimiento Personal (en adelante PCP); en la que la resultante numérica de la evaluación EPPO, 360 grados y por competencias alimente la PCP para todas las personas por niveles y funciones. La PCP requiere de un desarrollo tecnológico e informático que administre uno o más responsables de Administración del Desempeño, consolidando a través de ella: 
La reprogramación neurolingüísticamente que se requiere, aquella competencia y/o actitud que se haya identificado como brecha del desempeño y su nivel de impacto para la organización; así como su engranaje con sociedad, comunidad, y familia. En base a un análisis de la situación con el trabajador a través de una comunicación directa o equipo de intervención para la salud integral.

Ejemplo. Emprendedores con resiliencia, personas con pasión por lo hacenen todas sus esferas de interacción humana.

La utilización de diversos medios para las facilitaciones : e-learning, talleres presenciales, videos, incubadoras internas de competencias, así como atención del equipo de salud integral (bio-psico-socio-cultural).

La creación, en conjunto entre jefe y colaborador, de un plan de acción que sirva para monitorear en las reuniones informales de evaluación y que tenga una frecuencia mensual o bimensual.

La determinación de evidencia/s del producto a ser entregado, en beneficio de la organización, en beneficio del trabajador, de la familia y en los casos que sea medible de la comunidad.

La evaluación del producto de la PCP por parte del jefe y empleado, con asesoramiento del especialista de Administración del Desempeño.

El registro del cuadro de indicadores de gestión y su comportamiento (ascenso o descenso).

\section{CONCLUSIONES}

El valor de la Gestión del Manejo como sistema (conformado por políticas, normas, método, pilares, prácticas de secuelas, red interna para comunicación continua sobre funcionamiento y planes de acción, proceso de evaluación del funcionamiento, formato de evaluación, instructivo) y la aplicación del procedimiento EPPO radica en el impulso de una cultura hacia el elevado funcionamiento, que se evidencia por el espíritu competitivo y emprendedor de sus miembros en los diversos niveles, conjuntos, y responsabilidades. 
Alexander Véliz Montero

El procedimiento EPPO es útil al plasmarse un desarrollo de toda la administración parte de fines, metas estratégicamente predeterminadas, a partir del primer grado Gerencial hacia el grado inmediato inferior y de esta forma consecutivamente; lo cual se identificó como el mecanismo de cascada descendente que posibilita alinearse con lo estratégico, al proyecto de negocios, a las necesidades de los consumidores internos y/o externos, donde cada trabajador conoce sobre: las expectativas de su manejo, las pruebas conseguir, su proceso y cronograma de evaluación de cumplimiento de metas formal e informal, sus requerimientos a causa de los consumidores, las prácticas actitudinales tanto de jefes como de trabajadores, la construcción y desarrollo de su proyecto de incremento biopsico-socio-cultural y el engranaje de su administración en la organización.

La Gestión del Manejo es un sistema que ha proyectado la Dirección, y opera si el grado jerárquico sospecha firmemente y practica día a día los principios y valores de la organización. Se realiza con el esencial soporte de la Organización Estratégica y la Comunicación continua del funcionamiento como de todo lo estratégico. De esta forma es como el individuo comprometido con su papel y excede en sus resultados La vivencia de utilizar evaluaciones en el trabajo permitió detectar que es la Subjetividad el primordial inconveniente en Evaluaciones del Manejo. Inconveniente que perjudica a las/os evaluadas/os produciendo falta de pertenencia e insatisfacción por injusticia e inequidad lo que implica que se destruya por medio del procedimiento EPPO aplicado estricta y técnicamente.

Utilizar el desempeño laboral, con el criterio de 360 grados, con una base a Competencias Laborales, aporta a la administración humana de las empresas un efecto positivo pues se fomenta la autodirección, más grande colaboración del trabajador en su aumento y mejoramiento constante del manejo entre otros puntos de vista. Ir organizando e identificando de forma sencilla las pruebas del cumplimiento de metas laborales y particulares que fortalezcan a sus miembros como agentes de cambio cuya esencia sea alejarse de sus regiones de bienestar hacia el incremento personal y social así como aceptar como propios, valores y principios organizacionales.

Según la práctica profesional, se evalúa el funcionamiento por medio de un proceso y en menor medida por un sistema, de forma independiente de esto, la fortuna es la realidad de un lugar en el quehacer donde cabe la estimulación para la más grande productividad y el aumento de los individuos. No se divorcia de la administración, lo existente es un 
más grande trabajo que debería ser consistente. La iniciativa de producir y regir una Plataforma de Incremento Personal (PCP), posibilita a los directivos y asesores de la Gestión del Manejo caminar por una cultura competitiva y humana, que supone la alineación de los sistemas y procesos a lo estratégico aumentando los índices productivos creando una cadena espiritual de costo, que va a estimular al trabajador a devolver lo bueno que obtiene en la empresa a su familia, a sus equipos, y sociedad.

\section{BIBLIOGRAFÍA}

Byers y Rue. (2006). Administración de Recursos Humanos. México: Ed. Interamericana.

Chiavenato, I. (2004). Gestión del Talento Humano. Bogotá- Colombia: McGRAWHILL INTERAMERICANCA, S.A.

Cornejo, M. A. (5 de enero de 2014). Video La Pasión del Ser empresario. Obtenido de https://www.youtube.com/watch?v=g-J-YN31njU

Diana Martínez, María Zapata. (2007). Estado del Arte de Evaluación del Desempeño. Intellectum, intellectum.unisabana.edu.co 29pag.

Espinosa, J. G. (2011). calificación de Méritos. Evaluación de Competencias Laborales. México: Trillas.

Instituto Politécnico Nacional. (2011). Reporte Final. Nueva Epoca, Año 3 Volúmen 1 Número 5.

Kasuga, C. (30 de Noviembre de 2012). Video Conferencia de Como ser un líder, Como Manejar un Negocio Y Como Convertirse en Empresario Exitoso. .

Puchol, Luis. (2000). Cara y Cruz de la Evaluación del Desempeño. En L. Puchol, Casos y Supuestos en Dirección y Gestión de Recursos Humanos (págs. 120-123). Madrid. España: Diaz de Santos.

Puchol, Luis. (2003). Dirección y Gestión de Recursos Humanos. Madrid: Editorial Díaz de Santo, S.A.

Richard Swanson; Elwood Holton. (2002). Resultados. Cómo evaluar el desempeño, el aprendizaje y la percepción en las organizaciones. México: Oxford University Press México. 
Sanders, D. J. (2009). Conclusiones. En D. J. Sanders, Hecho para Servir. Cómo impulsar el desempeño de sus empleados de la línea frontal. (págs. 149-151). México: McGraw Hill.

Valencia, J. R. (2002). Administración Moderna de Personal. México: Ed. Thomson, sexta edición, p.16.

Valencia, M. E. (2005). Las Competencias Laborales: La estrategia laboral para la competitividad de las organizaciones? https://www.icesi.edu.co/revistas/index.php/estudios_gerenciales/article/view/170 /html, No. 96. 